\title{
"NO HAY ESCUELA PÚBLICA SIN WIFI". ESCOLARIZACIONES DE ESTUDIANTES CON DISCAPACIDAD INTELECTUAL EN TIEMPOS DE COVID-19 EN LA REGIÓN METROPOLITANA DE BUENOS AIRES
}

\section{"There isn't Public School without Wifi". Schooling of Students with Intellectual Disabilities in Times of Covid-19 in the Metropolitan Region of Buenos Aires}

Cintia Schwamberger

Universidad de General San Martín.

Laboratorio de Investigación en Ciencias Humanas-CONICET. Argentina cintiaschwamberger@gmail.com

Recepción: 10 de septiembre de 2020

Aceptación: 2 de marzo de 2021

Resumen: El COVID-19 expuso las desigualdades educativas y sociales, en general, más específicamente cuando nos referimos a procesos de escolarización de estudiantes con discapacidad intelectual en escuelas de educación especial emplazadas en contextos de pobreza urbana de la Región Metropolitana de Buenos Aires. En este informe, mediante un trabajo de investigación cualitativo describimos las prácticas docentes, a través de entrevistas en profundidad, desarrolladas por maestras de apoyo a la inclusión y docentes de escuelas especiales en tiempo de confinamiento. A partir de lo relevado sostenemos que el derecho a la educación de los y las estudiantes con discapacidad dependió exclusivamente de aquello que escuelas y docentes garantizaron por sus propios medios. Los primeros análisis muestran las condiciones materiales precarias en que se desarrolló la escolaridad e impactaron de modo directo en los procesos de enseñanza y aprendizaje de los y las estudiantes.

Palabras Clave: pandemia; desigualdad; escuela; discapacidad intelectual. 


\section{“NO HAY ESCUELA PÚBLICA SIN WIFI”. ESCOLARIZACIONES DE ESTUDIANTES CON DISCAPACIDAD INTELECTUAL EN TIEMPOS DE COVID-19 EN LA REGIÓN METROPOLITANA DE BUENOS AIRES C. SCHWAMBERGER}

AвSTRACT: COVID-19 exposed educational and social inequalities globally that are exacerbated in the global south more specifically when we refer to the schooling processes of students with intellectual disabilities in special education schools located in contexts of urban poverty in the Metropolitan Region of Buenos Aires. In this report, through a qualitative research work, we describe the teaching practices, through indepth interviews with teachers of support for inclusion and teachers of special schools, deployed in time of confinement. Based on the findings, we maintain that the right to education of students with disabilities depended exclusively on what schools and teachers guaranteed by their own means. The first analyzes show the precarious material conditions in which schooling was developed and had a direct impact on the teaching and learning processes of the students.

KEYWORDS: pandemic; inequality; school; intellectual disability.

\section{Introducción}

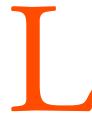

A PANDEMIA COVID-19 situó a los sujetos en general pero, en particular, a las personas con discapacidad en un estado de excepción (Agamben et al., 2020). En esa excepcionalidad se profundizaron las desigualdades y vulneraron más derechos (Grech, 2016; Palacios, 2020). Distintas reflexiones ahondaron en esta cuestión (Agamben et al., 2020; Giroux y Proasi, 2020). Sin embargo, dejaron de lado el impacto incurrido en las personas con discapacidad (PCD). De Sousa Santos (2020) fue quien se aventuró y propuso que las PCD han sido históricamente víctimas del capitalismo, el colonialismo, el patriarcado y el capacitismo. Esto a partir de una ideología de normalidad (Yarza y Vain, 2020), que define y divide aquellos cuerpos capaces de los que supuestamente no lo son (McRuer, 2006). Bajo esta perspectiva, las personas con discapacidad intelectual, en adelante PCDI, experimentan diversas barreras que limitan su acceso y participación social, exacerbando sus déficits y potenciando las desigualdades que viven.

En los países del sur global (Yarza y Vain, 2020), la pandemia dibujó un mapa de las barreras que transitan hacia las PCD, más aún cuando nos referimos al cierre de las instituciones educativas y la brecha digital existente (Cáceres-Muñoz et al., 2020; Dussel et al., 2020). La estrategia gubernamental promovió alternativas pedagógicas a través de plataformas, programas televisivos y contactos asincrónicos mediados por las tecnologías. Este proceso de “domiciliación” (Dussel et al., 2020, p. 337), irrumpió en los hogares de muy diversos modos, que, cuando los recursos no son los adecuados, la educación, más que un derecho, se convirtió en un privilegio. Lo anterior se recrudeció en la Región Metropolitana de Buenos Aires (RMBA), espacio marcado por la pobreza estructural (Grinberg, 2020), conectividad inexistente, condiciones habitacionales precarias, contextos no accesibles, ausencia de espacios de trabajo-estudio, falta de acceso a la salud, entre otras máximas que recorrieron el cotidiano escolar y social (Rockwell, 2018). Por tanto, importa centrarnos en las prácticas docentes desarrolladas en una escuela de educación especial emplazada en esta región que se encarga de los procesos de escolarización de PCDI. 
“NO HAY ESCUELA PÚBLICA SIN WIFI”. ESCOLARIZACIONES DE ESTUDIANTES CON DISCAPACIDAD INTELECTUAL EN TIEMPOS DE COVID-19 EN LA REGIÓN METROPOLITANA DE BUENOS AIRES C. SCHWAMBERGER

Como mencionamos, el plan de continuidad pedagógica se basó en propuestas tecnológicas con transmisión de contenidos curriculares. Escuelas y docentes, en un breve lapso, reorganizaron sus actividades y formas de conexión ante la medida de cierre (Cáceres-Muñoz et al., 2020). Desplegaron estrategias para llevar la escuela a los hogares y desde allí construyeron canales alternativos de comunicación y apoyos para garantizar el derecho a la educación. Entonces, ¿ cómo sostener la escuela cuando las políticas públicas no cubren las necesidades básicas del colectivo? (CEPAL, 2020; Unicef, 2020).

En tiempos de pandemia, sostener la presencialidad y el derecho a la educación mediante tecnologías se transformó en una de las barreras más fuertes y evidentes (Casquete-Tamayo, 2020; Dussel et al., 2020). Al respecto, la Secretaría de Evaluación e Información Educativa (SINIE, 2020) estableció una encuesta nacional sobre la escolaridad. Las PCD han sido relegadas de este estudio aun cuando representan el 2,9 $\%$ de los contagios positivos del país y su letalidad es un $7 \%$ más elevada que la del resto de la población. El instrumento comprobó que 3 de cada 10 hogares no poseen acceso fijo a Internet, el $27 \%$ accede por celular y el $3 \%$ no posee conexión alguna. Las dificultades en la señal son recurrentes y el $53 \%$ de los hogares no cuenta con portátiles para uso educativo. Asimismo, un $79 \%$ de docentes indicó limitaciones de conectividad y un $66 \%$ identificó problemas con el equipamiento. Con respecto a los vínculos, 9 de cada 10 docentes tuvieron contacto con sus estudiantes al menos una vez por semana, el resto dos o tres veces por mes o nunca tuvo contacto.

Estos datos evidencian la desigualdad de acceso a los recursos que impulsó, a escuelas y docentes, a intervenir de manera focalizada en las comunidades para intentar reducir la brecha digital y educativa (Ferreyra y Díaz, 2009; Maggio et al., 2014). En lo que respecta a estudiantes con discapacidad intelectual estas barreras se acentuaron con mayor intensidad (Baluarte-Carbajal, 2019; Emerson y Hatton, 2014). Investigaciones recientes (Casquete-Tamayo, 2020; Huete, 2020; Navas et al., 2020; Meaney-Davis et al., 2020) analizaron el impacto del confinamiento y demostraron el déficit gubernamental de las medidas implementadas para proteger al colectivo. En similar dirección, organizaciones civiles en Argentina-como REDI (Red por los Derechos de las Personas con Discapacidad)- encabezaron reclamos para garantizar derechos vulnerados, establecidos por la Convención Internacional de los Derechos de las Personas con Discapacidad (2006), la Ley de Educación Nacional n. 26.206 (2006) y la Resolución n. ${ }^{\circ}$ 311/16 del Consejo Federal, que afirman el derecho a la educación en igualdad de condiciones que el resto de la población. Ante esta vulneración se sumaron las sugerencias realizadas por la CEPAL (2020) y Unicef (2020), para revertir los procesos excluyentes que se exacerban cuando la discapacidad se cruza con la pobreza.

A partir de la ausencia de políticas efectivas, nos encontramos con actores escolares que debieron responder para garantizar esos derechos. Por lo tanto, son las docentes como refirió la directora de la escuela las que "cuando llegan los materiales, los bajan a sus celulares, los adaptan para sus estudiantes, los imprimen y, después, una vez por semana o cada quince días los entregamos a cada familia, junto con las viandas de alimentos si es que se pueden acercar a la escuela”. Entre estas condiciones de in- 
“NO HAY ESCUELA PÚBLICA SIN WIFI”. ESCOLARIZACIONES DE ESTUDIANTES CON DISCAPACIDAD INTELECTUAL EN TIEMPOS DE COVID-19 EN LA REGIÓN METROPOLITANA DE BUENOS AIRES

C. SCHWAMBERGER

dividualidad, soledad y garantía del derecho (Unicef, 2020), se dirimieron las distintas prácticas desplegadas por las docentes de esta escuela, que consiguieron, una vez más, sostenerse en pie, y que son eje de este informe.

\section{Metodología}

\subsection{Sobre el método}

Desde un enfoque cualitativo (De Seena, 2015), describimos las prácticas docentes, mediante entrevistas en profundidad, de una escuela especial emplazada en contextos de pobreza urbana de la RMBA. Son docentes de una escuela de gestión pública, de jornada completa, que se encarga de las trayectorias escolares (Brisciolli, 2016) de 499 estudiantes con discapacidad intelectual. El $51 \%$ de ellos transitan su escolaridad incluidos en escuelas de educación regular (Res 311/16) y el $49 \%$ restante permanece en la sede de la escuela especial. A su vez, el $60 \%$ de la matrícula escolar vive en los barrios más empobrecidos de la localidad, denominados villas miseria. Acercarnos de manera densa (Geertz, 2003) a las prácticas docentes permite comprender el estado de situación de los procesos de escolarización en tiempos de aislamiento.

\subsection{Participantes}

La muestra de este estudio es intencional, en el que participaron 10 docentes mujeres, de 35 a 50 años de edad, 6 de ellas maestras de apoyo a la inclusión (MAI) y 4 docentes de la escuela especial, que acompañan trayectorias escolares tanto al interior de la escuela como en las escuelas de educación regular.

\subsection{Instrumento y recolección de datos}

El instrumento seleccionado fue la entrevista en profundidad (De Seena, 2015), que se construyó a partir de un guion de 10 preguntas abiertas, junto a un diario de campo. Los ejes y categorías de abordaje fueron: 1) la conectividad, 2) los materiales y recursos utilizados para el desarrollo de las actividades, 3) los apoyos implementados, 4) la mediación docente-estudiante y 5) la intervención familiar. Se buscó indagar respecto a las posibilidades de conexión y acceso a recursos tecnológicos para el desarrollo de las clases virtuales, cuáles fueron los apoyos implementados, cómo se estableció el contacto con los y las estudiantes, qué recursos utilizaron y cómo se estableció el vínculo con las familias en este tiempo.

Las entrevistas se desarrollaron, entre abril y agosto del 2020, mediante plataformas tecnológicas. Si bien se organizó a través de un guión pautado, este último se propuso como un dispositivo flexible que fue variando el orden de las preguntas en los distintos encuentros. Esto permitió abrir historias, profundizar relatos y retomar 
“NO HAY ESCUELA PÚBLICA SIN WIFI”. ESCOLARIZACIONES DE ESTUDIANTES CON DISCAPACIDAD INTELECTUAL EN TIEMPOS DE COVID-19 EN LA REGIÓN METROPOLITANA DE BUENOS AIRES

C. SCHWAMBERGER

distintas prácticas que se analizaron conjuntamente. La duración se pautó con las entrevistadas y duró aproximadamente dos horas. Asimismo, se contó con el consentimiento informado para garantizar confidencialidad en cada entrevista. Se nominó a cada una de ellas por el cargo ejercido seguido de un número.

\subsection{Análisis de la información}

El diseño y el análisis de la información se realizaron a partir del método comparativo constante (Glaser y Strauss, 1967). En primer lugar, se realizó una lectura intensiva de la transcripción de las entrevistas y del diario de campo que las acompañaba. Seguidamente, se codificó y categorizó la información, a través de un sistema abierto en el que se establecieron familias de categorías y subtemas relacionados con los ejes de análisis del informe. El procesamiento de datos se elaboró mediante la utilización del programa de análisis cualitativo Atlas Ti, que permite manejar grandes volúmenes de información hasta la saturación de categorías. Los primeros resultados se agruparon en tres grandes áreas que se complementan mutuamente: conectividad, materiales y apoyos.

\section{Resultados preliminares y discusiones}

\subsection{Sobre la conectividad}

A partir de los datos relevados, el $90 \%$ de las docentes empleó recursos propios para garantizar clases virtuales y actividades impresas en cuadernillos. Un $75 \%$ de ellas manifestaron que sus estudiantes no realizaron las actividades entregadas. Con respecto a los vínculos, el $80 \%$ sostuvo un contacto semanal por medio de los grupos de WhatsApp. Un 10 \% solo se comunicó con la familia y el 10 \% restante no logró mantener contacto con sus estudiantes por falta de conectividad. Esto muestra que la promesa de la tecnología como superadora de desigualdades quedó en entredicho, así, también, lo refirió la docente 1 en el siguiente relato:

En mi casa solo tenemos una computadora que la usa mi hijo para estar con las clases virtuales. Las clases las preparo durante el tiempo que él no la utiliza, me las comparto a mi celular por el Whatsapp Web, las descargo y así desde el celular las envío al grupo que tengo de estudiantes de la mañana. Muchos de ellos no me responden o no ven el archivo que les mando. (Entrevista, docente 1, mayo de 2020)

A partir del anterior relato, el trabajo se complejizó en mayor intensidad cuando los recursos disponibles fueron insuficientes y se debió recurrir al celular para realizar actividades escolares como laborales propias. A ello, se sumaron los problemas de conexión existentes en la región: 


\section{“NO HAY ESCUELA PÚBLICA SIN WIFI”. ESCOLARIZACIONES DE ESTUDIANTES CON DISCAPACIDAD INTELECTUAL EN TIEMPOS DE COVID-19 EN LA REGIÓN METROPOLITANA DE BUENOS AIRES

Llamé como cinco veces a que me vengan a solucionar el problema de internet. Sin internet qué escuela pública estamos garantizando. La escuela pública hoy es gracias al Wifi que pagamos las docentes de nuestro bolsillo. (Entrevista, Docente 1, mayo de 2020)

La escuela es escuela gracias al wifi que las docentes pagaron de su bolsillo. Frase contundente que da cuenta de la precariedad en que se transitó este tiempo de escolaridad. La poca conectividad o su ausencia total no es exclusiva del estudiantado, sino que, como afirma la docente 1, debe reclamar para que solucionen el problema porque internet se convirtió en el insumo básico por excelencia para garantizar la continuidad pedagógica. En similar dirección, la docente 2 enunciaba:

Hoy internet es básico, se volvió tan básico como entregar las viandas de comida. Tengo el servicio de internet $\mathrm{X}$, uno de los más caros que hay en el país. Como ahora estamos todos en casa, la señal no resiste. Ya reclamé para que vengan a solucionarme el problema. Por más que pagues el mejor servicio de internet no tenés garantía de nada. (Entrevista docente 2, abril 2020)

Internet se volvió esencial, mencionó al igual que la docente 1 , y necesario para que exista escuela. El mayor problema es que, ante tal demanda, el servicio colapsó. En esta situación "estar conectado" se volvió, como afirma la docente 2, una necesidad primordial, precariamente garantizada.

Otra subcategoría analizada fue la conexión de los y las estudiantes:

Tengo en el grupo 17 chicos, la mayoría usa celular, pero cambian el chip, ya que no tienen internet en sus hogares. ¿Así pretendo que si le mando algo por chat descargue la tarea y me la vuelva a enviar? Consumen todos los datos con bajar una tarea por semana. Es imposible que se conecten. Con algunos me comunico con las madres, ya que es su único teléfono, para avisarles que tal día a tal hora vamos a estar entregando los alimentos y materiales en la escuela. (Entrevista docente 5, marzo 2020)

El uso de un único celular familiar, para lo escolar y lo cotidiano, transformó a la educación en una batalla titánica en sí y, aún más, cuando este recurso fue vital para acceder a los materiales y viandas que entregaba la escuela.

\subsection{Sobre los materiales}

La institución organizó cuadrillas que recorrieron distintos puntos de la localidad para entregar alimentos y materiales impresos preparados por docentes para cada grupo escolar:

Estoy preparando los cuadernillos para el grupo, de lo que mandan del gobierno no nos sirve nada, algunas imágenes o videos, pero después no hay ninguna actividad pensada para nuestros pibes. Tenemos que agarrar el cuadernillo y adaptarlo e imprimirlo 
“NO HAY ESCUELA PÚBLICA SIN WIFI”. ESCOLARIZACIONES DE ESTUDIANTES CON DISCAPACIDAD INTELECTUAL EN TIEMPOS DE COVID-19 EN LA REGIÓN METROPOLITANA DE BUENOS AIRES

C. SCHWAMBERGER

para mandárselo a cada uno de los chicos cuando la escuela entrega la mercadería. Nunca vuelven los cuadernillos, tampoco sabemos si los pueden hacer. Son familias con muchas necesidades y la verdad que nos importa en este contexto no solo que aprenda algo de lo que le mandamos, sino que también tenga alimentos y algún sostén que pueda llegar de la escuela. (Entrevista docente 4, abril de 2020)

Los materiales enviados por el Gobierno nacional debieron adecuarlos, porque no fueron pensados para PCDI, a partir de criterios de accesibilidad: ausencia de material en braille, lengua de señas o español sencillo (García et al., 2015). Situación que obstaculizó aún más la tarea docente. Acerca de la dificultad en los apoyos una MAI nos comentó cuando debía entregar materiales para sus estudiantes incluidos en las escuelas regulares:

Los profes mandan las actividades a sus estudiantes, nunca logramos que nos manden para realizar los ajustes necesarios en conjunto. Como tengo 13 estudiantes a mi cargo realizo todas las mañanas tres videollamadas grupales, separadas por niveles y materias. Lo que no me resulta sencillo y me provoca más trabajo es no solo configurar la actividad de manera solitaria para cada estudiante, sino que también, al dar la clase de manera personalizada, estamos volviendo a "integrar". Entonces, muchas veces me siento la maestra particular de mis 13 estudiantes como si estuviera en la especial y no una MAI que acompaña a los y las docentes de la escuela común. (Entrevista docente MAI 8, junio de 2020)

No contar con los materiales anticipadamente o diagramarlos en conjunto, situación recurrente e incluso prepandemia, fue la MAI quien debió accesibilizar las propuestas y garantizó apoyos específicos separados del grupo escolar, rememorando procesos integracionistas (Arnaiz-Sánchez et al., 2016), superados política y jurídicamente, que resurgen en estos tiempos. En efecto, la docente preparó la actividad, realizó videollamadas y logró desplegar contenidos y explicaciones que debieron ser realizados por los profesores de la escuela secundaria.

En los relatos que recuperamos se evidenciaron múltiples barreras, muchas de ellas existentes en tiempos prepandémicos. Barreras tecnológicas, de comunicación, conectividad e insuficientes apoyos que no fueron saldados por los programas gubernamentales. Empero ello, fueron compensados por las acciones que realizaron las docentes (Huete, 2020). Ocuparse de cada uno de sus estudiantes y familias, en este tiempo, no solo se volvió clave para garantizar la escolaridad y el derecho a la educación, sino, también, la escuela y sus docentes se ocuparon de cuestiones básicas como la entrega de alimentos y viandas.

El objetivo de este informe fue analizar algunas prácticas docentes desplegadas en la escuela. Se detectaron categorías claves respecto a los recursos, la conectividad y la no accesibilidad de los materiales. Los gobiernos intervinieron mediante políticas compensatorias (Ferrante, 2018) a los sectores más desfavorecidos, sin lograr eliminar la sombra de la exclusión. Los enunciados docentes demostraron que no alcanzó con asistir una vez a la semana a entregar cuadernillos o alimentos ya que, en condiciones de desconexión, la educación se tornó aún más desigual. 
“NO HAY ESCUELA PÚBLICA SIN WIFI”. ESCOLARIZACIONES DE ESTUDIANTES CON DISCAPACIDAD INTELECTUAL EN TIEMPOS DE COVID-19 EN LA REGIÓN METROPOLITANA DE BUENOS AIRES C. SCHWAMBERGER

En situaciones de hacinamiento, sin espacios propicios y sin un plato de comida es preciso preguntarse por los aprendizajes alcanzados. En condiciones de desigualdad y pobreza, los procesos de enseñanza-aprendizaje se vieron obstaculizados, más aún cuando nos referimos a estudiantes con discapacidad intelectual. Sin embargo, docentes y directivos no dejaron de sostener espacios de conexión y de encuentro para que algo de la escolaridad ocurra.

\section{Conclusiones}

La pandemia expuso el poder del neoliberalismo en toda su crueldad y recrudeció las desigualdades, maximizando sus lemas individualistas y excluyentes (Giroux y Proasi, 2020). Actualmente, se diagraman protocolos de regreso a las escuelas, particularmente para aquellos que accedieron de manera desigual a los contenidos curriculares. El regreso a la presencialidad sucede sin cuestionar las condiciones estructurales de precariedad en que estudiantes viven y transitaron la pandemia. Ante la ausencia de responsabilidades del sistema educativo y falta de inversión, para educar(se) en condiciones dignas, el problema recae y recayó en cada uno. Estudiantes y docentes debieron per se educar(se) en condiciones totalmente desiguales. Esto ocurrió en la escuela especial a la que hicimos referencia y en muchas de las escuelas de la región, caracterizadas por una materialidad precaria que potencia los obstáculos aún más, cuando se entrecruza con la pobreza y la discapacidad intelectual. Sin embargo, la escuela, todas las escuelas, una vez más, respondieron ante la exigente demanda y lograron que algo de lo común y lo público allí sucediera (Dusell et al., 2020).

Las instituciones se ocuparon de ese vacío gubernamental con los recursos disponibles y con los que no, como la falta de cuadernillos accesibles para PCDI, la entrega de alimentos o ayuda económica a las familias, entre otras prácticas desplegadas. La escuela se encargó de los rostros olvidados, que han quedado por fuera de las políticas públicas, a la deriva de mayores exclusiones (Grech, 2016). Empero ello, como sostuvimos, fue la escuela y sus docentes, una vez más, quienes garantizaron el derecho a la educación que, de no ser por el trabajo colectivo, tanto en las instituciones como en solitario desde sus hogares, los procesos de escolarización de estudiantes con discapacidad intelectual no hubieran sido posibles. A partir de aquí, surgen nuevos interrogantes respecto de los aprendizajes adquiridos, en tiempos de confinamiento, desde la perspectiva de estudiantes con discapacidad.

\section{Referencias bibliográficas}

Agamben, G., Zizec, S. y Nancy, J. (2020). Sopa de Wuhan: pensamiento contemporáneo en tiempos de pandemia. Aspo. Disponible en: https://www.elextremosur.com/files/content/23/23684/sopa-de-wuhan.pdf 
“NO HAY ESCUELA PÚBLICA SIN WIFI”. ESCOLARIZACIONES DE ESTUDIANTES CON DISCAPACIDAD INTELECTUAL EN TIEMPOS DE COVID-19 EN LA REGIÓN METROPOLITANA DE BUENOS AIRES C. SCHWAMBERGER

Arnaiz-Sánchez, P., Escarbajal Frutos, A., Guirao lavela, J. M. y Martínez Abellán, R. (2016). Building inclusive processes for school improvement: a case study. Journal of Research in Special Educational Needs, 16, 290-294. doi:10.1111/1471-3802.12291

Baluarte-Carbajal, A. (2019). Factores asociados a la calidad de vida en personas con discapacidad intelectual interdisciplinaria. Centro Interamericano de Investigaciones Psicológicas y Ciencias Afines, 36(1), 187-202. https://doi.org/10.16888/interd.2019.36.1.13

Briscioli, B. (2016). La incidencia de las condiciones de escolarización del nivel secundario en la construcción de las trayectorias escolares. Perfiles Educativos, 38(154), 134-153.

Cáceres-Muñoz, J., Jiménez Hernández, A. S. y Martín-Sánchez, M. (2020). Cierre de escuelas y desigualdad socioeducativa en tiempos del Covid-19. Una investigación exploratoria en clave internacional. Revista Internacional de Educación para la Justicia Social, 9(3), 199-221. https://doi.org/10.15366/riejs2020.9.3.011

Casquete-Tamayo, E. J. (2020). Vulnerabilidad ante el Covid-19 de la población infantil con discapacidad. Revista Científica Hallazgos21, 5(2), 171-184. Recuperado de http://revistas. pucese.edu.ec/hallazgos21/

Cepal. (2020). Sobre la evolución y los efectos de la pandemia del Covid-19 en América Latina y el Caribe. Disponible en: https://repositorio.cepal.org/bitstream/handle/11362/45491/ S2000300_es.pdf,jsessionid=73A44C40E6534E74312F1B6985c6952C? sequence=1

De SENA, A. (2015). Caminos cualitativos: aportes para la investigación en ciencias sociales. Ciccus.

De Sousa Santos, B. (2020). La cruel pedagogía del virus. Akal.

Dussel, I., Ferrante, P. y Pulfer, D. (2020). Pensar la educación en tiempos de pandemia: entre la emergencia, el compromiso y la espera. UNIPE.

Emerson, E. y Hatton, C. (2014). Health inequalities and people with intellectual disabilities. Cambridge University Press.

FERRANTE, C. (2018). 'Machetear': surviving disability through mendicity in the north of Chile. Alter. European Journal of Disability Research, 12(1), 26-40.

Ferreira, M. y Díaz, E. (2009). Discapacidad, exclusión social y nuevas tecnologías de la información. Política y Sociedad, 46(1 y 2), 235-258.

Fondo De Las Naciones Unidas Para La Infancia. Unicef. (2020). COVID-19 niños, niñas y adolescentes con discapacidad en el contexto de la pandemia. Argentina. Recuperado de http://www.msal.gob.ar/images/stories/bes/graficos/0000001858cnt-covid-19_niniescon-discapacidad-contexto-pandemia.pdf

García, C., Heredia, M., Reznik, L. y Rusler, V. (2015). La accesibilidad como derecho. Desafíos en torno a nuevas formas de habitar la Universidad. Espacios de Crítica y Producción, 51, 41-56. Disponible en: http://revistascientificas.filo.uba.ar/index.php/espacios/article/ view/1869

Geertz, C. (2003). La interpretación de las culturas. Gedisa.

Giroux, H. y Proasi, L. (2020). La pandemia del Covid-19 está exponiendo la plaga del neoliberalismo. Praxis Educativa, 24(2), 1-13. https://dx.doi.org/10.19137/praxiseducativa-2020-240202

Glaser, B. y Strauss, A. (1967). The discovery of Grounded. Theory strategies for qualitative research. Aldine Publishing Comp.

Grech, S. (2016). Disability in the Global South. The Critical Handbook. Springer.

Grinberg, S. (2020). Etnografía, biopolítica y colonialidad. Genealogías de la precariedad urbana en la Región Metropolitana de Buenos Aires. Tabula Rasa, 34, 19-39. https://doi. org/10.25058/20112742.n34.02 
“NO HAY ESCUELA PÚBLICA SIN WIFI”. ESCOLARIZACIONES DE ESTUDIANTES CON DISCAPACIDAD INTELECTUAL EN TIEMPOS DE COVID-19 EN LA REGIÓN METROPOLITANA DE BUENOS AIRES

Huete, A. (2020). Pandemia y discapacidad. Lecciones a propósito del confinamiento. Revista Española de Discapacidad, 8(I), 203-207.

Maggio, M., Lion, C. y Peroso, M. V. (2014). Las prácticas de la enseñanza recreadas en los escenarios de alta disposición tecnológica. Polifonías, año III, 5, 101-127.

Mcruer, R. (2006). Crip theory: cultural signs of queerness and disability. New York University Press.

Meaney-Davis, J., Lee, H. y Corby, N. (2020). The impacts of Covid-19 on people with disabilities. Disability Inclusion Helpdeskn, 35, 11-33.

Navas, P., Verdugo, M. Á., Amor, A. M., Crespo, M. y Martínez, S. (2020). COVID-19 y discapacidades intelectuales y del desarrollo: impacto del confinamiento desde la perspectiva de las personas, sus familiares y los profesionales y organizaciones que prestan apoyo. Plena inclusión y Universidad de Salamanca.

Organización De Las Naciones Unidas (Onu). (2006). Convención Internacional sobre los Derechos de las Personas con Discapacidad. Recuperado de: https://www.un.org/disabilities/documents/convention/convoptprot-s.pdf

Palacios, A. (2020). Discapacidad y derecho a la igualdad en tiempos de pandemia. Pensar, 25(4), 1-14.

Rockwell, E. (2018). Vivir entre escuelas: relatos y presencias (Antología Esencial). Clacso.

Yarza, A. y VaIn, P. (2020). Discapacidad y pandemia. Viejas y nuevas normalidades bajo sospecha. Clacso. 